Revista Colombiana de Obstetricia y Ginecología Vol. 57 No. 3 • 2006 • (163-170)

INVESTIGACIÓN ORIGINAL

\title{
VALIDEZ DEL CURETAJE ENDOCERVICAL EN LA DETECCIÓN DE LESIONES NO SOSPECHADAS DURANTE EL ESTUDIO COLPOSCÓPICO DE PACIENTES CON CITOLOGÍA ANORMAL ESCAMOSA
}

\author{
Efficacy of endocervical curettage in \\ colposcopic assessment of patients having \\ abinormal squamous pap smear \\ Antonio González-Mazuelo, M.D.* \\ Recibido: junio 8/06 - Revisado: agosto 15/06 - Aceptado: agosto 28/06
}

\section{RESUMEN}

Objetivo: el uso del curetaje endocervical (CEC) durante la colposcopia está actualmente en medio de una importante controversia: se duda de su utilidad diagnóstica en las pacientes con sospecha de anormalidad escamosa en la citología cervicovaginal y el enfoque clásico del problema, compara el resultado del mismo contra el estudio histológico del cuello, utilizado por diversos autores, no ha ayudado a resolver el dilema. La presente investigación da un nuevo enfoque al incluir en el análisis la biopsia dirigida y orientado a la búsqueda de lesiones no sospechadas por el examinador, a fin de mejorar la seguridad del diagnóstico colposcópico.

Metodología: se tomaron los datos de 306 mujeres con citología anormal escamosa y CEC durante el estudio colposcópico, el cual se comparó con el resultado histopatológico de la totalidad del cuello uterino en la búsqueda de lesiones no sospechadas en la biopsia dirigida y analizadas respecto a la

* Médico Ginecólogo y Obstetra de la Universidad de Antioquia. Profesor asociado, Universidad Pontificia Bolivariana. Ginecólogo de la Empresa Social del Estado Metrosalud de Medellín. Dirección: calle 1 sur \#43C161, apartamento 310, Medellín, Colombia.

Correo electrónico: antoniogonzalez@epm.net.co satisfacción o no de la colposcopia y el resultado de la citología.

Resultados: en general se encontraron valores de sensibilidad bajos, mientras que el valor predictivo negativo y la especificidad fueron altos.

Conclusión: el CEC realizado bajo indicaciones precisas, de acuerdo al tipo de colposcopia y anormalidad citológica, es de utilidad y mejora la seguridad del diagnóstico coloscópico.

Palabras clave: neoplasia intraepitelial del cuello uterino; curetaje endocervical; biopsia; sensibilidad y especificidad.

\section{SUMMARY}

Objective: using endocervical curettage during colposcopy is currently being questioned; its usefulness as a diagnostic tool in patients having suspected squamous changes in pap smear is controversial. The classical approach to this problem adopted by many authors has yielded little help; it consists of matching histological results from the cervix with the problem itself. The current research provides a new approach by including biopsy orientated towards searching for lesions which were not suspected during examination; this was aimed 
at trying to improve the safety of colposcopy when used as a diagnostic tool.

Methodology: results from 306 women having abnormal pap smear and endocervical curettage during colposcopic evaluation were thus compared to histopathological results from cervical specimens in the search for non-suspicious lesions during guided biopsy and analysed according to colposcopy and pap smear results.

Results: overall, sensitivity was low while specificity and negative predictive values were high.

Conclusion: the outcomes led to the conclusion that endocervical curettage performed in line with strict indications, according to the type of colposcopy and pap smear abnormality, is of great help and improves the safety of colposcopic diagnosis.

Key words: cervical intraepithelial neoplasia, curettage, biopsy, sensitivity and specificity.

\section{INTRODUCCIÓN}

El examen mediante colposcopia y biopsia dirigida es el procedimiento universalmente aceptado en la actualidad para la evaluación inicial de las mujeres con citología anormal ${ }^{1}$ y el curetaje endocervical (CEC) hace parte del mismo como método complementario.

Algunas de las indicaciones para su realización están claras como en el estudio de las alteraciones citológicas glandulares y para decidir una reconización cuando uno previo ha reportado márgenes comprometidos en el tratamiento de la neoplasia intraepitelial cervical (NIC), ${ }^{2}$ pero su uso en el estudio colposcópico de las alteraciones escamosas siempre ha sido polémico. En la literatura mundial existen diferentes opiniones sobre su utilidad sin que hasta ahora se haya logrado un consenso al respecto. ${ }^{3-5} \mathrm{Se}$ reconocen diversas posiciones, desde las extremas de los que abogan su uso rutinario en todas las colposcopias ${ }^{6}$ hasta los que lo consideran como innecesario, pasando por quienes piensan que se hace o no dependiendo de ciertas consideraciones y por indicación. ${ }^{7}$ Quienes defienden su uso sistemático piensan que así se previenen lesiones ocultas, y los de opinión contraria piensan que una colposcopia satisfactoria no lo requiere pues en ellas las lesiones ocultas son raras y las colposcopias insatisfactorias necesitan siempre un procedimiento excisional diagnóstico (PED).

Una colposcopia es satisfactoria si la unión metaplásico-columnar (UMC) es completamente visible y por ende todas las lesiones de la zona de transformación y las que se introducen en el canal pueden ser totalmente evaluadas. Cuando estos requisitos no se cumplen la colposcopia es insatisfactoria. Este concepto, supuestamente, ayuda a prevenir errores de diagnóstico que se generarían al dejar de detectar carcinomas escamosos ocultos o lesiones glandulares (adenocarcinomas) localizados en la portio o en el canal endocervical.

El CEC se introdujo como conducta rutinaria durante la colposcopia ${ }^{8,9}$ en la década de los años 80, cuando se hacía uso extenso de los métodos destructivos locales en el tratamiento de la NIC y a partir de un reporte de Townsend y Reichert ${ }^{10}$ sobre una serie de casos de carcinoma de cuello uterino después de crioterapia. El CEC ayudaría a prevenir esta situación provocada por el colposcopista al no reconocer adecuadamente la UMC o al no identificar dichas lesiones invasivas. ${ }^{11}$

Sin embargo, el papel del CEC en las colposcopias satisfactorias es muy limitado ya que las lesiones no están usualmente al alcance del instrumento y además, la crioterapia y la vaporización láser han dejado de usarse como tratamiento de la NIC y fueron sustituidas por técnicas excisionales que permiten una segunda evaluación histológica del cuello uterino y la detección de las escasas lesiones desapercibidas.

La indicación del CEC más aceptada es en la colposcopia insatisfactoria. Una de las razones en que el colposcopista falla en identificar un carcinoma invasivo es su presencia en el canal endocervical no visualizado durante el examen; si durante la colposcopia, en estas circunstancias, se hace un CEC, el resultado negativo o normal del mismo proporcionaría la evidencia de que no hay 
una lesión displásica mayor o infiltrante ocultas. El uso rutinario de instrumental apropiado para la visualización del canal endocervical (espéculo endocervival o microhisteroscopia) ha reducido la frecuencia de colposcopia insatisfactoria limitando la necesidad del CEC.

El CEC tiene limitaciones de sensibilidad que reducen su valor dependiendo de la cureta usada, la fortaleza del raspado, el muestreo completo de la longitud y circunferencia del cilindro endocervical y la retención de parte de la muestra por el moco., ${ }^{5,12}$ Además, su especificidad puede comprometerse por la contaminación desde la portio con las lesiones visibles, incrementando los falsos positivos y el número de PED. Por otra parte, hay estudios que demuestran que el CEC disminuye la cantidad de PED en un porcentaje considerable de $\operatorname{casos}^{13}$ en contraposición con otros informes donde el CEC positivo fue la mayor indicación para realizarlos. ${ }^{14}$

Clásicamente los estudios sobre la importancia diagnóstica del CEC se hicieron aislándolo del resultado de la biopsia dirigida y comparándolo con el estudio histológico completo del cuello uterino obtenido por histerectomía o procedimiento excisional (resección con asa de radiofrecuencia o conización); los resultados fueron equívocos y a veces contradictorios. Las estadísticas son igualmente variables en cuanto a la sensibilidad y especificidad del mismo, así como lo referente a los falsos positivos y negativos; Igualmente contradictorios han sido los trabajos que han querido limitar la importancia del CEC en la toma de decisiones terapéuticas. ${ }^{15}$

La asociación americana de patología cervical y colposcopia (ASCCP) recientemente publicó una guía sobre el uso del CEC durante la colposcopia en el estudio de las alteraciones escamosas en la citología teniendo como criterio la severidad de la misma y el tipo de colposcopia. ${ }^{16}$ En ellas no se recomienda su uso en colposcopia satisfactoria y citologías con células escamosas atípicas (ASC) o lesiones de bajo grado (LSIL) si hay lesiones visibles, y un uso discrecional en citologías con lesiones de alto grado (HSIL) si se puede hacer una adecuada valoración endocervical. Se recomienda el curetaje endocervical en las pacientes con colposcopias insatisfactorias, en citologías con células escamosas atípicas o lesiones de bajo grado porque si la biopsia dirigida y el CEC son negativos se evitaría un procedimiento excisional diagnóstico y en las pacientes con citologías con lesiones de alto grado, en caso de obtener una biopsia dirigida y CEC negativos, para decidir la revisión de todo el material antes de planear el PED.

De este modo, se realizó un estudio para evaluar la validez diagnóstica del curetaje endocervical en las pacientes investigadas con colposcopia y biopsia dirigida por citología anormal escamosa. Se compararon las que tuvieron curetaje endocervical durante la misma y las que no lo tuvieron. Todas fueron sometidas a procedimientos quirúrgicos con resultados histológicos de la totalidad del cuello uterino a fin de correlacionar este resultado con las biopsias dirigidas y ver el papel que cumplió el CEC en la detección de lesiones mayores ocultas. En este estudio se consideró el CEC como un examen complementario que ayudaría a mejorar la precisión de la biopsia dirigida y dependiendo de su resultado, la decisión de profundizar más en el estudio del cuello antes del procedimiento terapéutico.

\section{MATERIALES Y MÉTODOS}

Diseño: estudio de exactitud diagnóstica ensamblado sobre un estudio de corte transversal. El patrón de oro estuvo dado por la presencia de una lesión oculta no sospechada por la biopsia dirigida en el resultado histológico de la totalidad del cuello uterino obtenido por procedimiento quirúrgico.

Población: 3.533 pacientes de estrato socioeconómico bajo a quienes se hizo colposcopia por presentar citología cervico vaginal anormal, procedimientos que fueron tomados de la base de la Empresa Social del Estado METROSALUD, institución de salud adscrita a la alcaldía de Medellín, entre los meses de mayo de 1991 a diciembre de 2001.

Criterios de inclusión: información completa de los resultados de la biopsia dirigida, complementada o no con curetaje endocervical y del estudio 
anatomopatológico del cuello uterino obtenido por procedimiento excisional o histerectomía. El muestreo fue secuencial por conveniencia.

Procedimiento: los resultados de la biopsia dirigida se compararon con los de la patología definitiva del cuello uterino. Se establecieron los casos positivos y negativos para una lesión mayor no diagnosticada durante el examen colposcópico; este resultado fue cruzado en una tabla de dos por dos con el obtenido en el curetaje endocervical según fue positivo o negativo.

En forma simultánea se obtuvieron las prevalencias de lesiones mayores no diagnosticadas en las pacientes que no fueron sometidas a curetaje endocervical según el tipo de colposcopia, satisfactoria o no, para compararlas con las del grupo estudiado y determinar si había diferencias.

Definición de términos: se define como colposcopia satisfactoria aquella donde se logra la visualización completa de la UMC y colposcopia insatisfactoria si no se visualizó totalmente, a criterio del especialista examinador. CEC positivo, el que el patólogo reporta como muestra adecuada y con evidencia de neoplasia, CEC negativo aquel que el patólogo reporta como adecuado y negativo para neoplasia. Se define como caso positivo para una lesión mayor no diagnosticada (oculta) durante el examen colposcópico al resultado anatomopatológico del cérvix obtenido por procedimiento excisional o histerectomía que histológicamente reportó grados mayores que el resultado de la biopsia dirigida así: si el estudio del cérvix encontró carcinoma o adenocarcinoma y la biopsia dirigida respectiva cualquier lesión NIC, VPH o cervicitis, o si el estudio del cérvix reportó NIC II-III y la biopsia dirigida respectiva solo cervicitis, VPH o NIC I. Se definió como caso negativo para una lesión mayor no diagnosticada (oculta) durante el examen colposcópico al estudio anatomopatológico del cérvix obtenido por procedimiento excisional o histerectomía cuyo reporte histológico fue igual o menor al de la biopsia dirigida respectiva.
Se definió como error terapéutico cualquier paciente insuficientemente tratado con un procedimiento primario a consecuencia del deficiente diagnóstico hecho durante la colposcopia y su biopsia dirigida.

Análisis: se diseñó una encuesta para la obtención de los datos de las historias clínicas, que se trasladó al programa Epi - info 6.04 para su análisis y obtención de resultados. Se calculó la prevalencia de lesiones mayores no diagnosticadas en cada grupo, la sensibilidad, la especificidad, y los valores predictivos negativo y positivo con sus respectivos intervalos de confianza del 95\%, el porcentaje de concordancia y el coeficiente de probabilidad positivo del curetaje endocervical en la detección de esas lesiones. Se hizo un análisis estratificado de acuerdo a si la colposcopia era satisfactoria o no y por el resultado de la citología según tuvieran alteraciones menores (ASC-US o LSIL) o mayores (ASC - H, HSIL o cáncer).

Esta investigación cumple con los requisitos éticos según la Resolución 008430 de 1993 (Título II, Capítulo 1, Artículo 11) del Ministerio de Salud de Colombia y fue avalada por el Comité de Ética de la facultad de medicina de la Universidad Pontificia Bolivariana.

\section{RESULTADOS}

Las 306 pacientes con curetaje endocervical en quienes se logró obtener la información necesaria para la presente investigación, tenían un promedio de edad de 41 años, rango entre 19 y 77 años. La base de datos reportó 534 pacientes sin curetaje endocervical con reporte histológico de la totalidad del cuello uterino obtenido por procedimiento quirúrgico que sirvieron de grupo de control para la prevalencia de lesiones ocultas.

Entre los 33 casos de colposcopia satisfactoria y citologías menores, solo una paciente con biopsia dirigida como cervicitis reportó una lesión mayor (NIC II); su CEC fue negativo (tabla 1). La sensibilidad del CEC fue 0\%, especificidad de 89,4\% (74,5 - 96), el valor predictivo negativo (VPN) de 95,2\% 


\begin{tabular}{|l|c|c|c|}
\multicolumn{3}{|c|}{$\begin{array}{c}\text { Tabla } \\
\text { 1. Resultados del curetaje } \\
\text { endocervical en colposcopias } \\
\text { satisfactorias y citologías menores. }\end{array}$} \\
\hline & $\begin{array}{c}\text { Caso } \\
\text { positivo }\end{array}$ & $\begin{array}{c}\text { Caso } \\
\text { negativo }\end{array}$ & Total \\
\hline CEC positivo & 0 & 3 & 3 \\
\hline CEC negativo & 1 & 29 & 30 \\
\hline Total & 1 & 32 & 33 \\
\hline
\end{tabular}

CEC: curetaje endocervical.

\begin{tabular}{|l|c|c|c|}
\hline \multicolumn{3}{|c|}{$\begin{array}{c}\text { Tabla 2. Resultados del curetaje } \\
\text { endocervical en colposcopias } \\
\text { satisfactorias y citologías mayores. }\end{array}$} \\
\hline & $\begin{array}{c}\text { Caso } \\
\text { positivo }\end{array}$ & $\begin{array}{c}\text { Caso } \\
\text { negativo }\end{array}$ & Total \\
\hline CEC positivo & 2 & 26 & 28 \\
\hline CEC negativo & 2 & 125 & 127 \\
\hline Total & 4 & 151 & 155 \\
\hline
\end{tabular}

CEC: curetaje endocervical.

$(81,5$ - 98,9) valor predictivo positivo (VPP) de $12,5 \%(1,3-60,4)$ y el porcentaje de concordancia $87,9 \%$; el cociente de probabilidad positivo fue de 2,35 (intervalo 0,17 - 31,6). En este grupo no se presentaron errores terapéuticos.

Entre los 155 casos de colposcopia satisfactoria y citologías mayores, cuatro pacientes con biopsias dirigidas como NIC II-III reportaron lesiones mayores ocultas (un adenocarcinoma invasivo, 2 carcinomas microinvasivos IA1 y un carcinoma IB) (tabla 2); la sensibilidad del CEC fue 50\% (15 - 85), especificidad de 82,8\% (76 - 88), el VPN de 98,4\% (94,4 - 99,6), el VPP de 7,1\% (2 - 22,6) y el porcentaje de concordancia $81,9 \%$; el cociente de probabilidad positivo fue de 2,9 (intervalo 1,02-8,22). En este grupo se presentó un error terapéutico por violación del protocolo (evitable) al no proceder con PED luego de un CEC positivo: la paciente se llevó a histerectomía simple sin PED y tenía un carcinoma IB insuficientemente tratado.

En general, independiente del resultado de la citología, si la colposcopia fue satisfactoria, un 16,5\% de los CEC fueron positivos con una sensibilidad de 40\% (11,8 - 76,9), especificidad de 84,1\% (78,2

\begin{tabular}{|l|c|c|c|}
\multicolumn{4}{|c|}{$\begin{array}{c}\text { Tabla 3. Resultado del curetaje } \\
\text { endocervical en colposcopias } \\
\text { no satisfactorias y citologías menores. }\end{array}$} \\
\hline & $\begin{array}{c}\text { Caso } \\
\text { positivo }\end{array}$ & $\begin{array}{c}\text { Caso } \\
\text { negativo }\end{array}$ & Total \\
\hline CEC positivo & 1 & 6 & 7 \\
\hline CEC negativo & 0 & 9 & 9 \\
\hline Total & 1 & 15 & 16 \\
\hline
\end{tabular}

CEC: curetaje endocervical.

\begin{tabular}{|c|c|c|c|}
\hline \multicolumn{4}{|c|}{$\begin{array}{c}\text { Tabla } \\
\text { 4. Resultado del curetaje } \\
\text { endocervical en colposcopias } \\
\text { no satisfactorias y citologías mayores. }\end{array}$} \\
\hline & $\begin{array}{c}\text { Caso } \\
\text { positivo }\end{array}$ & $\begin{array}{c}\text { Caso } \\
\text { negativo }\end{array}$ & Total \\
\hline CEC positivo & 7 & 38 & 45 \\
\hline CEC negativo & 7 & 50 & 57 \\
\hline Total & 14 & 88 & 102 \\
\hline
\end{tabular}

CEC: curetaje endocervical.

- 88,7), el VPN de 98,1\% (94,5 - 99,3), el VPP de $6,45 \%(1,8-20,7)$ y concordancia de $83 \%$. El cociente de probabilidad positivo fue de 2,5 (intervalo $0,82-7,76)$.

Entre los 16 casos de colposcopia insatisfactoria y citologías menores, solo una paciente con biopsia dirigida como NIC III reportó lesión mayor (un carcinoma microinvasivo IA2) tuvo el CEC positivo y se procedió adecuadamente con PED (tabla 3); la sensibilidad del CEC fue 100\% (19,5 - 100), especificidad de 59,4\% (35,9-79,2), el VPN de 95\% $(65,5$ - 99,5), el VPP de $18,8 \%(4,5-53,3)$ y el porcentaje de concordancia de $62,5 \%$. El cociente de probabilidad positivo fue de 1,84 $(0,68-4,99)$. En este grupo no se presentaron errores terapéuticos.

Entre los 102 casos de colposcopia insatisfactoria y citologías mayores, siete con biopsias dirigidas como cervicitis reportaron lesiones mayores (siete NIC II - III) y siete con biopsias dirigidas como NIC II - III reportaron lesiones mayores (2 adenocarcinomas invasivos, 2 carcinomas microinvasivos IA1 y 3 microinvasivos IA2) (tabla 4); la sensibilidad del CEC fue $50 \%(26,8-73,2)$, especificidad de 56,8\% (46,4 - 66,7), el VPN de 87,1\% (76,8 - 93,9) el VPP de 
$15,1 \%(7,7-28,8)$ y el porcentaje de concordancia 55,9 . El cociente de probabilidad positivo fue de 1,15 (intervalo 0,65-2,6). Este grupo presentó dos errores terapéuticos en pacientes sometidas a histerectomía simple con carcinoma IA2 y adenocarcinoma invasivo; en una se confió en el resultado negativo del CEC y la otra por violación de protocolo (evitable) pues su CEC dio positivo y no se hizo PED. De proceder, basados en el CEC negativo en los otros casos, los errores terapéuticos hubieran aumentado a siete.

En general, independiente del resultado de la citología, si la colposcopia fue insatisfactoria, un 44\% de los CEC fueron positivos con una sensibilidad de 53,3\% (30,1 - 75,2), especificidad de 57,3\% (47,6 - 66,4), el VPN de 89,4\% (79,7 - 94,8), el VPP de $15.4 \%(8-27,5)$ y el porcentaje de concordancia $56,8 \%$. El cociente de probabilidad positivo fue de $1,24(0,74-2,10)$.

La prevalencia de lesiones mayores no diagnosticadas en las colposcopias satisfactorias que tuvieron CEC fue de 2,66\% (5/188) y no hay diferencia significativa con el grupo de control sin CEC de 516 pacientes, que reportó 11 lesiones mayores no diagnosticadas y una prevalencia de 2,13\% $\left(\mathrm{X}^{2}\right.$ : $0,32, \mathrm{p}>0,10)$. La prevalencia de lesiones mayores no diagnosticadas en las colposcopias insatisfactorias que tuvieron CEC fue de 12,7\% (15/118) y no hay diferencia significativa con el grupo de control sin CEC de 18 pacientes que reportó dos lesiones mayores no diagnosticadas y una prevalencia de $11,1 \%$ $\left(\mathrm{X}^{2}: 0,43 \mathrm{p}>0,10\right)$.

La prevalencia total (incluyendo el grupo de estudio y los controles) de lesiones histológicas ocultas no diagnosticadas en las coloscopias cuando la citología era menor fue de 1,46\% (3/205), 1,08\% $(2 / 184)$ en colposcopia satisfactoria y 4,76\% (1/21) en las insatisfactorias. Cuando la citología era mayor, la prevalencia de lesiones histológicas ocultas no diagnosticadas en la colposcopia fue 4,72\% (30/635), $2,69 \%(14 / 520)$ en colposcopia satisfactoria y $13,9 \%$ $(16 / 115)$ en las insatisfactorias.

El VPP tiene relación directa con la prevalencia de lesiones ocultas y este aumenta al aumentar aquella así: VPP de 6,45\% con prevalencia de 2,66\% de las colposcopias satisfactorias a 15,4\% de VPP con prevalencia de 12,7\% de las colposcopias insatisfactorias.

\section{DISCUSIÓN}

En los trabajos realizados en los últimos años con el objetivo de evaluar la utilidad del CEC en la planificación del tratamiento de las pacientes con enfermedad cervical se ha considerado como innecesario. ${ }^{17,18}$ Este estudio contiene un enfoque nuevo en la valoración del papel diagnóstico del CEC durante la colposcopia al medir y comparar la prevalencia de las lesiones mayores no diagnosticadas (ocultas) en cada circunstancia y la capacidad de la prueba en su detección, pero no de forma separada, sino integrada al resultado de la biopsia dirigida.

A pesar de no tener una determinación previa del tamaño muestral, la muestra es mayor que la de otros estudios similares previos. ${ }^{19}$

En este reporte, la prevalencia de lesiones ocultas en la colposcopia satisfactoria cuando la citología es menor es muy baja (1\%); el curetaje endocervical no aportó en su diagnóstico probablemente al no estar localizada en la zona de raspado. Ninguna de las 33 pacientes portaba una carcinoma o adenocarcinoma oculto. Los falsos positivos $(9,4 \%)$ incrementarían el número de procedimientos excisionales diagnósticos. Parece aceptable la recomendación de la ASCCP de no usarlo en estos casos. ${ }^{16}$ Otros estudios han propuesto la posibilidad de omitir el CEC en los casos de ASC-US / LSIL y colposcopia satisfactoria y normal. ${ }^{3}$

La prevalencia de lesiones ocultas en la colposcopia satisfactoria cuando la citología es mayor es el doble que las citologías menores pero sigue siendo baja (2,7\%). En esta serie de 155 casos hubo lesiones significativas ocultas, el CEC contribuyó a prevenir el 50\% de las mismas y su alto VPN $(98,4 \%)$ daría tranquilidad en centros donde se recurre a la histe-rectomía simple como principal medio terapéutico y/o cuando no se tiene experiencia en colposcopia y es difícil definirla como 
satisfactoria. Los falsos positivos por contaminación de la muestra $(17,2 \%)$ incrementarían el número de PED en forma significativa. Parece aceptable la recomendación de la ASCCP de usarlo en estos casos en forma discrecional dependiendo de la valoración endocervical. ${ }^{16}$

La prevalencia de lesiones ocultas en la colposcopia insatisfactoria cuando la citología es menor es mas alta (4,7\%); el curetaje endocervical aportó el diagnóstico del único caso (carcinoma IA2). Lo más destacable sería su alto VPN que permite descartar lesiones ocultas sin necesidad de hacer PED, los cuales se reducen del 100\% al 43,7\% (solos los CEC positivos, 7/16); las 9 pacientes con CEC negativo no tenían lesiones mayores ocultas. Parece aceptable la recomendación de la ASCCP de usarlo en estos casos para evitar PED. ${ }^{16}$

La prevalencia de lesiones ocultas en la colposcopia insatisfactoria cuando la citología es mayor es seis veces más alta (13,9\%). En esta serie de 102 casos hubo lesiones significativas ocultas; el CEC contribuyó a prevenir el 50\% de los mismos con una sensibilidad similar a situaciones anteriores pero el VPN $(87,1 \%)$ es más bajo que en las otras circunstancias, dejando de detectar un número significativo de lesiones con riesgo de error terapéutico. De este hallazgo parece inferirse que no es aceptable la recomendación de la ASCCP de usar el CEC en estos casos para decidir cuales pacientes requieren un $\mathrm{PED}^{16}$ y por el contrario sería más seguro hacerlo en todos los casos. Ello indica que en pacientes con una citología mayor y una colposcopia insatisfactoria no es posible considerar un resultado negativo del CEC como ausencia de enfermedad oculta.

De los resultados de esta serie se destaca que la prevalencia de las lesiones ocultas se incrementa progresivamente con la severidad de la citología y la localización de la zona de transformación en el canal endocervical.

La prevalencia de lesiones ocultas en colposcopia satisfactoria es similar a lo reportado por Helmerhorst de 2,44\%; en cambio este autor informó el doble de prevalencia en colposcopia insatisfactoria $(22,2 \%){ }^{20}$

Desde otro enfoque, sin tener en cuenta la citología, en las pacientes con colposcopia satisfactoria, el CEC fue positivo en 16,5\% de los casos, muy similar a lo reportado en la literatura, que en promedio fue 10\%., Este fenómeno ocurre generalmente por contaminación de la muestra y rara vez por lesiones endocervicales o multicéntricas, o por la existencia de dos lesiones diferentes. ${ }^{21,22}$ Por el contrario, en las pacientes con colposcopia insatisfactoria, el CEC fue positivo en 44\% de los casos, muy similar a lo reportado por otros estudios, que en promedio fue $48 \%{ }^{23}$

Los resultados del presente estudio muestran que la sensibilidad tiene valores muy bajos similares al estudio de Andersen de 55\%. ${ }^{5}$ Un reporte chileno informa una cifra mayor, $75 \% .{ }^{9}$ En cambio el VPN fue importante en las pacientes con colposcopia satisfactoria, independiente del tipo de citología y en las insatisfactorias con citologías menores, similares al reporte de Helmerhorst del 99,4\%. ${ }^{20}$ Se puede concluir que en este tipo de pacientes la realización del CEC permite excluir, con alto margen de seguridad, las lesiones no sospechadas que inducen errores terapéuticos.

\section{REFERENCIAS}

1. Committee on Practice Bulletins - Gynecology. ACOG practice bulletin. Diagnosis and treatment of cervical carcinomas. Obstet Gynecol 2002;99:855-67.

2. Bidus MA, Elkas JC, Rodriguez M, Maxwell GL, Rose GS. The clinical utility of the diagnostic endocervical curettage. Clinical Obstet Ginecol 2005;48:202-8.

3. Wllliams DL, Dietrich C, McBroom J. Endocervical curettage when colposcopy examination is satisfactory and normal. Obstet Gynecol 2000;95:801-3.

4. Moniak CW, Kutzner S, Adam E, Harden J, Kaufman RH. Endocervical curettage in evaluation abnormal cervical cytology. J Reprod Med 2000;45:285-92.

5. Andersen W, Frierson H, Barber S, Tabbarah S, Taylor P, Underwood P. Sensitivity and specificity of endocervical curettage and the endocervical brush 
for the evaluation of the endocervical canal. Am J Obstet Gynecol 1988;159:702-7.

6. Dreyfus M, Baldauf JJ, Ritter J. Diagnostic value of endocervical curettage during colposcopy. Eur J Obstet Gynecol Reprod Biol 1996;64:101-4.

7. Ayoubi JM, Cayrol MH, Meddoun M, Benevent JB, Degoy J, Pons JC. Role of endocervical curettage in the screening for cervical cancer: Apropos of a series of 31 cases. Gynecol Obstet Fertil 2000;28:455-9.

8. National Comprehensive Cancer Network. Practice Guidelines in Oncology, 2004. Visitado 2004 Julio 30. Disponible en: http://www.nccn.org

9. Puente R, Hoffmann L, Castro A. Valor del legrado endocervical en el diagnóstico colposcópico de enfermedad de cuello uterino. Rev Chil Obstet Ginecol 1995;60:28-33.

10. Instituto Nacional de Cancerología. Pautas de tratamiento: Cáncer de Cuello Uterino en CD-ROM. Bogotá: El Instituto; 2002.

11. Massad LS, Collins YC. Using history and colposcopy to select women for endocervical curettage. Results from 2.287 cases. J Reprod Med 2003;48:1-6.

12. Donohue LR, Meriwether W. Colposcopy as a diagnostic tool of cervical neoplasias. Am J Obstet Gynecol 1972;113:107-10.

13. Wetrich DW. An analysis of the factors involvement in the colposcópic evaluation of 2194 patients with abnormal Papanicolaou smears. Am J Obstet Gynecol 1986;154:1339-49.

14. Moseley KR, Dinh TV, Hannigan EV, Dillard EA Jr, Yandell RB. Necessity of endocervical curettage in colposcopy. Am J Obstet Gynecol 1986;154:992-5.
15. Irving W, Flora S, Andersen W, Stoler M, Taylor P, Rice L. Endocervical curettage. Does it contribute to the management on patients with cervical cytology? J Reprod Med 2004;49:1-7.

16. Wright TC Jr, Cox JT, Massad LS, Twiggs LB, Wilkinson EJ; ASSP-Sponsored Consensus Conference. 2001 Consensus Guidelines for management of women with cervical cytological abnormalities. JAMA 2002;287:2120-9.

17.Fine DA, Feinstein GI, Sabella V. The pre-and postoperative value of endocervical curettage in the detection of cervical Intraepithelial neoplasia and invasive cervical cancer. Gynecol Oncol 1998;71:46-9.

18. Ferenczy A. Endocervical curettage has no place in the routine management of women with cervical intraepithelial neoplasia: debate. Clin Obstet Gynecol 1995;38:644-8.

19. Díaz CA, Aragón ME. Valor del curetaje endocervical en el diagnóstico de neoplasias cervicales: Instituto Materno Infantil de Bogotá, 1986-1988. Rev Colomb Obstet Ginecol 1991;42:209-17.

20. Helmerhorst TJ. Clinical significance of endocervical curettage as part of colposcopic evaluation. A review. Int J Gynecol Cancer 1992;2:256-62.

21. Hatch KD, Shinglenton HM, Orr JW Jr, Gore H, Soong SJ. Role of endocervical curettage in colposcopy. Obstet Gynecol 1995;65:403-8.

22. Tate KM, Strickland JL. A randomized controlled trial to evaluate the use of the endocervical brush after endocervical curettage. Obstet Gynecol 1997;90:715-7.

23. Dresher CW, Peters WA 3rd, Roberts JA. Contribution of endocervical curettage in evaluating abnormal cervical cytology. Obstet Gynecol 1983;62:343-7. 